\title{
Análisis comparativo de propuestas para la ordenación de la actividad extractiva de áridos en la isla de Tenerife
}

\author{
Germán Hernández-Durán(1), Julio César Arranz-González ${ }^{(2)}$ y Rogelio de la Vega-Panizo(3) \\ (1) INTERRA, Ingeniería y Recursos. Santo Domingo, 2, 38003, Sta. Cruz de Tenerife, España. \\ german@interra.es \\ (2) Departamento de Investigación en Recursos Geológicos, Instituto Geológico y Minero de España. \\ Ríos Rosas, 23, 28003 Madrid, España. \\ jc.arranz@igme.es \\ (3) ETS de Ingenieros de Minas y Energía. Universidad Politécnica de Madrid. Alenza, 4, 28003 Madrid, España \\ rogelio.delavega@upm.es
}

\begin{abstract}
RESUMEN
La Isla de Tenerife es un territorio con numerosos espacios naturales protegidos y otras áreas merecedoras de protección ambiental, como consecuencia de su riqueza en valores ecológicos y paisajísticos. De acuerdo con las actuales normas de planificación, la actividad extractiva de áridos está limitada a una serie de áreas denominadas ámbitos extractivos. La delimitación de estas zonas presenta algunos defectos e incoherencias, tanto desde el punto de vista ambiental como desde la consideración de la capacidad productiva de recursos mineros. Este trabajo muestra dos modelos de planificación ambiental aplicada a la actividad extractiva de áridos para la Isla de Tenerife, los cuales pueden servir como alternativas al modelo de planificación vigente: un modelo denominado zonificado y otro llamado zonificado-concentrado. Ambos modelos proporcionan mejoras en lo referente al respeto de los valores ambientales y parten del conocimiento de la distribución territorial de los materiales geológicos. Se concluye que el conocimiento de la distribución territorial de los recursos potenciales es clave para alcanzar la integración efectiva de la minería en la ordenación territorial, y la mejor forma posible para salvaguardar los recursos minerales frente a actividades que pueden comprometer su disponibilidad futura. Las metodologías aplicadas pueden hacerse extensivas a otros territorios, en particular insulares, en los que es muy posible que exista una problemática semejante a la existente en la Isla de Tenerife.
\end{abstract}

Palabras clave: Áridos, Canarias, minería, planificación territorial, recurso minero, Tenerife

\section{Comparative analysis of proposals for the planning of the aggregate industry on the island of Tenerife}

\author{
ABSTRACT
}

The island of Tenerife is a territory with numerous protected natural areas and other areas which also deserve environmental protection, due to their ecological and landscape richness and values. According to current planning regulations, the aggregate mining industry is limited to a number of areas called extractive zones. The delimitation of such areas has some flaws and inconsistencies from the environmental point of view and from the consideration of the productive capacity of mining resources. This paper shows two models for environmental planning applied to the extractive activity for aggregates for the island of Tenerife, which could serve as alternatives to the current planning model: a model called zoned and another designated as zone-concentrated. These models provide improvements as far as respect for environmental values are concerned and are based on knowledge of the spatial distribution of geological materials. The conclusion is that this knowledge of the distribution of the potential mineral resources in the territory is the key to achieving the effective integration of mining in land-use planning, and to safeguard in the best possible way the mineral resources from the activities that may compromise their future availability. The applied methods 
Hernández-Durán, G., et al., 2016. Análisis comparativo de propuestas para la... Boletín Geológico y Minero, 127 (4): $755-773$

could be extended to other territories, particularly islands, where it is very possible that there are similar problems to those that are occurring in Tenerife.

Keywords: aggregates, Canary Islands, land planning, mining, mining resource, Tenerife

\section{ABRIDGED ENGLISH VERSION}

\section{Introduction and methods}

Spatial planning applications for aggregate mining can be vital on an island territory, such as the island of Tenerife, where the supply conditions of sufficient quality and quantity can hardly be met through alternative means such as recycling or importing. Table 1 shows the main geological resources that currently cover the aggregate demand. Moreover, Tenerife is a territory with numerous protected natural areas and other areas which also deserve environmental protection, due to their environmental and landscape richness and values. It should be noted that the vast majority of the abandoned aggregate quarries that have been inventoried in Tenerife are unrestored. As a result, the territory is dotted with landscape scars of varying size. In addition, it is estimated that only $25 \%$ of the mining operations were authorized. These data shed light on the poor image of the sector and justify the current planning model, which can be considered as restrictive for mining. Today, the spatial planning of mining activity is regulated by the Decree 56/2011 of 4 March (BOC, 1011). According to the current planning regulations, the aggregate mining industry is limited to a number of areas called extractive zones (Fig. 1). This is a management model which can conceptually be referred to as a model of concentration. Tables 2 and 3 show some data related to the authorized extractive areas in the current land- use plan. The delimitation of the extractive zones of the existing planning model has some flaws and inconsistencies from the environmental point of view, and from the consideration of the productive capacity of mining resources. The lack of consideration for existing geological resources is a major flaw, since it means that aggregate resources remain at risk of sterilization (Stenestad y Sustrac, 1994; Langer, 2002) as a result of a hypothetical expansion of alternative land uses. This paper shows two models for environmental planning applied to the extractive activity for aggregates for the island of Tenerife, which could serve as alternatives to the current planning model: a model called a zoned model and another that can be designated as a zone-concentrated model.

For the effective integration of mining in spatial planning it is necessary to have detailed knowledge and to delimit those areas of land that have possibilities for the exploitation of mineral resources, and to assess their quantity and quality (Barettino et al., 2003). Therefore, the planning process began with the assessment of the existence and quality of the mineral resources, establishing three quality categories (Fig. 2). The analysis was performed by pooling the resources of major uses of aggregates: aggregates for construction (concrete, asphalt and mortar) aggregates for prefabricated pieces and industrial aggregates (pozzolans). To analyze the carrying capacity of the territory, from the territorial point of view, the extent of the impact that can be directly comparable to the value of the type of land use at risk of being affected was taken into account (ArranzGonzález et al., 2009). This premise served to exclude, in a first approximation, all the areas of greatest environmental or territorial value. Following the terminology used by IGME (1995) these would be areas with very low carrying capacity, derived from a high impact, regardless of the geological-mining aptitude. The location of exploitations was considered unacceptable in these areas, therefore mining activity should be prohibited. To set new levels of carrying capacity for the aggregate resource exploitation, other criteria called restrictive variables were taken into account. In all areas where these restrictive variables appeared, it was considered that the exploitation of mineral resources should be prohibited, except in exceptional cases. Following the terminology used by IGME (1995) this would be the areas with low carrying capacity. Then, another set of conditioning variables were considered, taking into account fragments of the territory in which there is a certain degree of incompatibility. These conditioning variables were used to identify areas where carrying capacity would be intermediate. Throughout the rest of the island territory, at the working scale used, it can be said that carrying capacity is high and the level of impact may be acceptable, with the subsequent rehabilitation of the land, so that extractive activity can be considered vocational, as long as there are quality resources.

\section{Results and discussion}

For the establishment of the proposals of mining planning in Tenerife, the geological aptitude was first considered, counting only the areas that were mapped within the categories of medium and high quality for the different types of use: aggregates for construction, aggregates for prefabricated pieces and industrial 
Hernández-Durán, G., et al., 2016. Análisis comparativo de propuestas para la... Boletín Geológico y Minero, 127 (4): $755-773$

aggregates. Two models of planning were also considered: zoned and zone-concentrated. According to the zoned model, the territory was divided into five categories, in which the activity should have a different degree of implementation in terms of its carrying capacity. Figure 3 outlines the method followed. The following types of surfaces were recognized (Fig. 4):

- Areas where extractive activity will be prohibited, including protection zones and areas initially recognized without resources or with low-quality resources.

- Areas where extractive activity will be prohibited, except in exceptional cases, defined on the basis of the presence of restrictive variables.

- Areas where extractive activity will be conditioned, defined on the basis of the presence of conditioning variables.

- The rest of the territory, where extractive activity can be allowed without conditions. Territorially, these are areas where there are suitable resources, without any restrictive or limiting conditions, having also been affected by previous mining activity. Activity in these areas could be developed without any conditions, except those imposed by legislation on mining, environment and safety.

To obtain the zone-concentrated model, the areas representing the two classes of higher carrying capacity were chosen, having regard to the existence of already exploited areas (Fig. 5), i.e., only those areas were included which were not recognized by both restrictive conditions or variables, and were also influenced by previous mining activity. In addition, only those areas were considered that had sufficient surface to form an extractive zone similar to those proposed by the current regulations. The extractive activity in these areas could be developed without any conditions, which are called areas of extractive activity allowed without conditions. The rest of the territory can be considered prohibited areas for extractive activity (Fig. 6). As with the current model, the island territory was divided into two categories, but after a more systematic examination of the distribution of mineral resources, and other environmental considerations that allow a rather conservative outcome. The extractive zones proposed for the different types of resources are shown in Table 4. Both models lead to improvements in relation to the respect of environmental values and are based on the knowledge of the spatial distribution of the geological resources. A use of more resources would thus be achieved, ensuring supply for a long time, despite the high level of environmental care. It is concluded that this knowledge in the territory is the key issue to achieve the effective integration of mining in land-use planning, and to safeguard in the best possible way the mineral resources from the activities that might compromise their future availability. The applied methods could be extended to other territories, particularly islands, where it is very possible that there are similar problems to those that occur in the island of Tenerife.

\section{Introducción}

El compromiso entre la extracción de los recursos geológicos y la protección ambiental debe ser alcanzado en el marco de una adecuada ordenación territorial, teniendo en cuenta las peculiaridades de la actividad minera (Barettino et al., 1994), de forma que un análisis de diferentes alternativas de localización permita seleccionar las más óptimas, considerando conjuntamente la mayor rentabilidad con el mínimo impacto posible (Barettino, 2002; Arranz González y Alberruche del Campo, 2008). Barettino (2002) subrayó que la planificación territorial aplicada a la minería es, por lógica, más adecuada para los recursos minerales que suelen presentarse con relativa abundancia en cuanto a su distribución geográfica, como ocurre con los materiales de construcción entendidos según los criterios de Kužvart (1984): los áridos naturales y de machaqueo, las calizas para fabricación de cemento, algunas clases de arcillas y las rocas ornamentales. La ordenación territorial enfocada a la explotación de áridos puede ser de vital importancia en un territorio insular. Este es el caso de la Isla de Tenerife, en la que el suministro en condiciones de calidad y cantidad suficiente difícilmente puede ser atendido mediante vías alternativas como el reciclaje o la importación. En Tenerife, además, la demanda de áridos está muy ligada al ejercicio de actividades clave en la economía insular, por lo que la explotación de los mismos tiene un carácter estratégico. Por otro lado, los valores naturales de Tenerife son en muchos aspectos excepcionales. El reconocimiento de tales valores se hace explícito en los 43 Espacios Naturales protegidos recogidos en el Decreto Legislativo 1/2000 de 8 de mayo (BOC, 2000), los cuales suponen un $48.6 \%$ de la superficie insular. Igualmente, mediante el Decreto 174/2009 (BOC, 2010), se declaran las Zonas Especiales de Conservación (ZEC) integrantes de la Red Natura 2000 en Canarias, reconociéndose 47 zonas en la isla de Tenerife, junto a 7 ZEPA (Zonas de Especial Protección para las Aves). También, mediante el Decreto legislativo 1/2000 (BOC, 2000), se definen y delimitan las denominadas "Áreas de Sensibilidad Ecológica", que se suman a todas las anteriores figuras de protección. No hay que olvidar además que existen otro tipo de elementos o usos del 
suelo limitantes o excluyentes de la minería o actividad extractiva, como son los elementos del patrimonio cultural, los desarrollos urbanísticos, los terrenos dedicados a la agricultura de alta rentabilidad, etc. En la actualidad, la planificación territorial de la actividad extractiva está regulada en la Revisión Parcial del Plan Insular de Ordenación de Tenerife, mediante Decreto 56/2011 (BOC, 2011). En esta revisión no sufren modificación las disposiciones normativas que en su día fueron incorporadas al Plan Insular de Ordenación de Tenerife (PIOT), aprobado mediante el Decreto 150/2002 (BOC, 2002). Según estas normas, las actividades mineras han de concentrarse en determinadas zonas, llamadas ámbitos extractivos, exceptuando aquellas explotaciones activas autorizadas con anterioridad a su promulgación. Para el resto del territorio, la minería queda prohibida, salvo en circunstancias excepcionales que se describen más abajo. Esta es una fórmula de planificación que pretende minimizar los problemas ambientales y paisajísticos asociados a explotaciones a cielo abierto, en un territorio en el que existe además abundante superficie protegida. Sin embargo, este modelo de ordenación presenta algunos problemas que tienen su origen en los criterios tenidos en cuenta a la hora de delimitar los ámbitos.

El trabajo que aquí se presenta se ha centrado en los recursos de áridos y fue desarrollado como parte de un proyecto en el que se abordó un diagnóstico de la situación actual de la actividad extractiva en Tenerife desde diversos enfoques: ambiental, legal, económico y estratégico (INTERRA, 2011). El objetivo fundamental es describir dos alternativas o modelos de planificación generados aplicando metodologías de ordenación minero-ambiental con la idea de que pudieran sustituir al modelo de ordenación de la actividad extractiva vigente. Fue necesario analizar la problemática minero-ambiental existente, teniendo en consideración todos los factores, tanto ambientales como extractivos, que han de intervenir en la cuantificación de las zonas con mayor potencial para la implantación de explotaciones, sin perder de vista los usos más habituales de los diferentes tipos de recursos mineros. En el análisis territorial se emplearon herramientas SIG, que permiten contemplar en su conjunto tanto la delimitación cartográfica de los recursos como las distintas figuras de planificación existentes.

\section{Algunos rasgos significativos de la minería de áridos en la Isla de Tenerife}

El consumo de áridos para la construcción y la obra pública está en Tenerife, como en otros lugares, relacionado con el incremento de la población y el desarrollo de infraestructuras. Además está muy ligado al principal sector productivo de la economía: el turismo, especialmente a las infraestructuras turísticas. Como ocurre con otros sectores económicos, la construcción ha mostrado una desaceleración en los últimos años. Es de esperar antes o después una reactivación de la actividad constructiva. En la Tabla 1 se detallan los principales recursos geológicos de los que se surte actualmente la demanda de áridos. La gran mayoría de los recursos geológicos de Tenerife son de origen volcánico. En Tenerife se aprovechan basalto y fonolita para obtener áridos de machaqueo, empleándose sobre todo para la fabricación de aglomerado asfáltico y hormigón. Otro recurso importante es la piedra pómez, compuesta por piroclastos de caída de tipo sálico que se presenta en fragmentos muy porosos, de muy baja densidad y de color claro. Sus principales usos son la fabricación de cemento puzolánico, ciertos usos agrícolas y la fabricación industrial de abrasivos.

\begin{tabular}{|l|l|}
\hline \multicolumn{1}{|c|}{ Litología / recursos geológicos } & \multicolumn{1}{c|}{ Usos posibles } \\
\hline Basalto y rocas intermedias & Áridos para construcción (hormigones, aglomerado asfáltico, escolleras) \\
\hline Fonolitas y traquitas & $\begin{array}{l}\text { Áridos para construcción (hormigones aglomerado asfáltico) } \\
\text { Roca ornamental (placas, baldosas, adoquines) }\end{array}$ \\
\hline $\begin{array}{l}\text { Depósitos aluviales (gravas y arenas de } \\
\text { barranco) }\end{array}$ & $\begin{array}{l}\text { Áridos para hormigones y aglomerado asfáltico } \\
\text { Arenas para morteros } \\
\text { Subbases de carretera }\end{array}$ \\
\hline $\begin{array}{l}\text { Piroclastos basálticos e intermedios } \\
\text { (lapilli o picón) }\end{array}$ & $\begin{array}{l}\text { Áridos para prefabricados (bloques y bovedillas) } \\
\text { Subbases de carretera }\end{array}$ \\
\hline Piroclastos sálicos (piedra pómez) & Áridos industriales y cementos puzolánicos \\
\hline
\end{tabular}

Tabla 1. Tipos de recursos geológicos utilizados para la obtención de áridos en la Isla deTenerife

Table 1. Types of geological resources used to obtain aggregates on the island of Tenerife. 
Hernández-Durán, G., et al., 2016. Análisis comparativo de propuestas para la... Boletín Geológico y Minero, 127 (4): $755-773$

También existen recursos de arenas y gravas de origen sedimentario, ligadas a la dinámica fluvial, que se asocian a cauces de barrancos antiguos o terrazas colgadas sobre los cauces. Su composición y granulometría es variada. Destacan los depósitos de los Barrancos de Güímar, el mayor yacimiento de estas características en Canarias. Su uso fundamental es el de árido para la fabricación de hormigones, arena para morteros, subbases de carreteras $y$, en menor medida, para fabricación de aglomerado asfáltico. El árido para uso en prefabricados proviene en su totalidad de canteras de picón (lapilli y arena que lo acompaña). Los áridos utilizados en la fabricación de morteros son mayoritariamente arenas de barranco y de machaqueo, junto a un pequeño porcentaje de importación. Por último existe un tipo de actividad extractiva un tanto especial, en el sentido de que no es nada habitual en otros territorios. Se trata de la explotación de tierra vegetal como insumo para la agricultura, la cual se limita a zonas muy concretas. Actualmente, en Tenerife, la producción de áridos no solamente proviene de canteras registradas o legales, sino también de plantas móviles que tratan excedentes de obras públicas y vaciados de solares, materiales reciclados de construcción y demolición, así como también materiales obtenidos en extracciones ilegales. Son importantes en cuanto a su producción y están en auge, pero no pueden sustituir a la producción de cantera a la hora de cubrir todas las necesidades. Estas plantas han surgido por dos motivos principales: la paralización de la explotación de áridos en la zona de los Barrancos de Güímar, y la existencia de infraestructuras que facilitaban la obtención de material de desmonte en obra pública. De hecho, la mayoría del árido empleado en la construcción de infraestructuras proviene de excedentes del movimiento de tierras de la obra civil. Aproximadamente la mitad del árido consumido se destina a la fabricación de hormigones. El resto se destina en un $18 \%$ a subbases y zahorras, $17 \%$ a prefabricados, $9 \%$ a mortero $y$, finalmente un $7 \%$ a la fabricación de aglomerado asfáltico (www.minetur.gob.es/energia/mineria/Estadistica/Paginas/ Consulta.aspx)

Analizando los documentos de base elaborados para la primera versión del PIOT (BOC, 2002) se obtuvo el inventario de explotaciones. La consulta de dichos documentos se vio facilitada por el Cabildo Insular de la Isla de Tenerife. Los perímetros de las explotaciones inventariadas fueron digitalizados, actualizando los contornos en base a orto-fotografías del año 2009. Del total de las 297 extracciones inventariadas en Tenerife, 28 están activas, 200 están abandonadas sin restaurar y el resto han sido remodeladas o sostienen un nuevo uso del suelo. El resultado es un territorio salpicado de cicatrices de mayor o menor tamaño que, bien por su forma o bien por su ubicación, dificultan la implantación de nuevos usos o actuaciones de restauración que permitan la integración paisajística en el entorno. Por otro lado, se estima que sólo un $25 \%$ de las explotaciones mineras fueron autorizadas. Estos datos ayudan a entender la mala imagen del sector $y$, en cierto modo, justifican el modelo de planificación vigente, el cual puede considerarse restrictivo para la actividad minera.

\section{Modelo de ordenación vigente: ámbitos extractivos}

Como se ha mencionado, en el modelo de ordenación territorial vigente se establece: "los usos minero-extractivos se han de asignar siempre a ámbitos acotados con carácter temporal, de forma que el ejercicio de las intervenciones asociadas a los mismos se limita al plazo que se establezca en la autorización $y$, finalizadas éstas, se debe proceder a la restauración de los terrenos para permitir el ejercicio de otros usos" (BOC, 2011). Así pues, la actividad extractiva ha de limitarse a determinadas zonas llamadas ámbitos extractivos (Fig. 1, Tabla 2), con dos excepciones: cuando tengan carácter artesanal, y para la extracción de piedra en bloques para obras públicas de interés insular. Se trata de un modelo de ordenación que conceptualmente puede denominarse modelo de concentración. La ordenación dentro de los ámbitos extractivos ha de establecerse mediante Planes Territoriales Parciales (PTP). En principio, las explotaciones de áridos en el resto del territorio se considerarán como usos a extinguir, y en ellas sólo serán autorizables las actividades de restauración, existiendo además medidas de carácter transitorio para las explotaciones activas. La delimitación de los ámbitos extractivos se hizo de acuerdo con los siguientes criterios (BOC, 2011):

- "Evitar actividades extractivas en zonas del territorio donde no se hubieran producido anteriormente, para limitar nuevos impactos y facilitar el tratamiento conjunto del territorio afectado

- Concentrar el ejercicio de la actividad extractiva en un número limitado de ámbitos, con una distribución equilibrada de la producción por recursos y zonas de la isla, evitando la dispersión de canteras por todo el territorio

- Compatibilizar los usos mineros con el resto de usos del territorio, de acuerdo al Modelo de Ordenación propuesto y a las previsiones de desarrollo en el tiempo de las diversas comarcas 


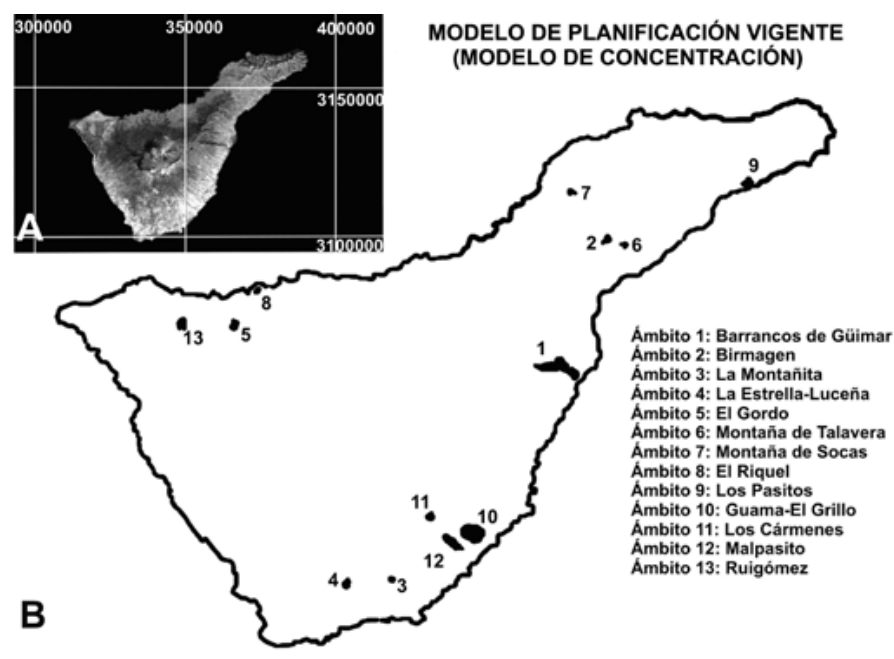

Figura 1. A: Malla UTM superpuesta a una imagen aérea de la Isla de Tenerife (descargada de la página web http://www2.ign.es/iberpix/visoriberpix/visorign.html). Las líneas del mallado están espaciadas $50 \mathrm{~km}$. Los números en blanco se corresponden con las coordenadas referidas al sistema de referencia REGCAN95/UTM (huso 28). B: Ámbitos extractivos establecidos en el modelo de ordenación territorial vigente (modificado de BOC, 2011).

Figure 1. A: UTM grid superimposed on an aerial photograph of the island of Tenerife (down-loaded from web page http://www2. ign.es/iberpix/visoriberpix/visorign.html). Grid lines are spaced 50 $\mathrm{km}$ apart. Numbers in white refer to UTM coordinates according to REGCAN95/UTM system (zone 28N). B: Extractive zones determined by the current land-use planning model (adapted from BOC, 2011).
- Garantizar la suficiente capacidad extractiva para la demanda previsible de cada recurso en el horizonte de programación del Plan, pero también permitir la viabilidad productiva del sector, evitando situaciones de rigidez de la oferta derivada de una excesiva limitación de los ámbitos extractivos".

Salvo el ámbito extractivo denominado Ruigómez, que alberga un área tradicional de extracción de tierra vegetal, empleada como material de préstamo para el desarrollo de cultivos de exportación, el resto de ámbitos habrán de producir la gran mayoría de los áridos consumidos en Tenerife a lo largo del horizonte temporal contemplado en el Plan (que no se especifica). Por lo que respecta a la situación de las canteras activas respecto a los ámbitos extractivos, todas las canteras de picón salvo una, están dentro de alguno de ellos. Las dos canteras de puzolanas también están dentro de ámbitos extractivos. Sin embargo, las dos canteras que funcionan en la actualidad para la producción de áridos para hormigones (el recurso más consumido) queda fuera de los ámbitos extractivos establecidos. Observando el número de ámbitos destinados a cada uso (Tabla 2), el número de ámbitos para suministrar áridos para hormigones es reducido (3), frente a los ámbitos de picón (6)

\begin{tabular}{|l|l|l|l|}
\hline \multicolumn{1}{|c|}{ Denominación } & \multicolumn{1}{|c|}{ Recurso } & \multicolumn{1}{c|}{ Municipio } & \multicolumn{1}{c|}{ Uso del recurso } \\
\hline Ámbito 1: Barrancos de Güímar & Gravas-arenas & Güímar & $\begin{array}{l}\text { Áridos para hormigones, aglomerados y } \\
\text { morteros }\end{array}$ \\
\hline Ámbito 2: Birmagen & Picón & El Rosario & Áridos para prefabricados \\
\hline Ámbito 3: La Montañita & Picón & Granadilla de Abona & Áridos para prefabricados \\
\hline Ámbito 4: La Estrella-Luceña & Picón & San Miguel & Áridos para prefabricados \\
\hline Ámbito 5: El Gordo & Picón & Icod de los Vinos & Áridos para prefabricados \\
\hline Ámbito 6: Montaña de Talavera & Picón & Santa Cruz de Tenerife & Áridos para prefabricados \\
\hline Ámbito 7: Montaña de Socas & Picón & Tacoronte & Áridos para prefabricados \\
\hline Ámbito 8: El Riquel & Piedra & Icod de los Vinos & $\begin{array}{l}\text { Áridos para hormigones, aglomerados y } \\
\text { morteros }\end{array}$ \\
\hline Ámbito 9: Los Pasitos & Piedra & Santa Cruz de Tenerife & $\begin{array}{l}\text { Áridos para hormigones, aglomerados y } \\
\text { morteros }\end{array}$ \\
\hline Ámbito 10: Guama-El Grillo & Puzolana & Arico & Cemento puzolánico y abrasivos \\
\hline Ámbito 11: Los Cármenes & Puzolana & Granadilla de Abona & Cemento puzolánico y abrasivos \\
\hline Ámbito 12: Malpasito & Puzolana & Granadilla de Abona & Cemento puzolánico y abrasivos \\
\hline Ámbito 13: Ruigómez & Tierra vegetal & ElTanque & Sustrato agrícola \\
\hline
\end{tabular}

Tabla 2. Ámbitos extractivos autorizados en el plan de ordenación vigente para la Isla deTenerife (modificado de BOC, 2011).

Table 2. Authorized extractive areas in the current land-use plan for the island of Tenerife (adapted from BOC, 2011). 
o los de puzolanas (3). El volumen de materiales puzolánicos que podría extraerse es equiparable al de áridos para prefabricados, cuando las necesidades reales son inferiores. Esto significa que el horizonte temporal que es capaz de cubrirse con los ámbitos es mucho mayor en el caso de las puzolanas que en el de áridos para hormigones. Sin embargo esto no significa que las reservas de los ámbitos proveedores de los áridos para hormigones sean insuficientes. La Tabla 3 muestra estimaciones temporales de capacidad de suministro de los ámbitos extractivos vigentes. Se ha determinado la demanda insular anual de recursos geológicos a partir del consumo medio de cemento de los años 2005 a 2009, con información facilitada por OFICEMEN (Agrupación de Fabricantes de Cemento de España). Los documentos de base del PIOT (BOC, 2002) establecían para la mayoría de los Ámbitos Extractivos un volumen de recurso aprovechable. Algunos de esos ámbitos disponen de estudios de mayor detalle elaborados para sus correspondientes documentos de ordenación, por lo que resultó conveniente actualizar las cifras de previsión del PIOT. Esa actualización de volúmenes, o volúmenes estimados, corresponden a los definidos en las alternativas escogidas en cada documento de Avance (INTERRA, 2011).

Es importante destacar que la delimitación de los ámbitos extractivos no se realiza después de un análisis sistemático del potencial geológico-minero de la isla. Este hecho constituye un defecto importante del modelo de planificación vigente, posibilitando que los recursos de áridos queden en situación de riesgo de esterilización derivado de la expansión de otros posibles usos del suelo.

Teóricamente, la concentración de la actividad en zonas donde ya existen explotaciones puede presentar ventajas desde el punto de vista ambiental, puesto que posibilita un tratamiento conjunto de los problemas ambientales y permite un desarrollo coordinado de los proyectos de restauración. Sin embargo, dos de los ámbitos incluyen sectores pertenecientes a figuras de protección ambiental: el ámbito de Los Pasitos dentro del Parque Rural de Anaga, y el ámbito de El Riquel, que incluye en su parte oriental a una Zona Especial de Conservación (ZEC). Los Pasitos tiene el grueso de sus recursos en la zona de intersección con el citado Parque Rural de Anaga. Con respecto al ámbito de El Riquel, existe un importante rechazo a la explotación minera por parte de la población. También es cierto que además de la delimitación de dichos ámbitos extractivos se contempla la necesidad de restaurar las áreas afectadas de modo que se favorezca una integración en estos entornos. No obstante, si se consideran otras variables importantes, como las áreas urbanas y de expansión urbana, las zonas de cauces y barrancos, la distancia respecto a zonas protegidas o el litoral, tendrá que ser el desarrollo de los PTP el que posibilite un grado de afección coherente con las normas de ordenación territorial, puesto que la norma sólo fija la delimitación externa de cada ámbito. La consideración de todos estos aspectos en cada ámbito podría suponer una reducción importante de la superficie útil en todos ellos.

En la Fig. 1 puede verse que, desde el punto de vista de la ubicación, los ámbitos extractivos tienen una buena localización como centros de producción. En cambio, al tratarse de zonas con derechos mineros adquiridos, es posible que se imposibilite en buena medida la aparición de nuevas empresas. Además, en aquellas zonas donde, con anterioridad a la delimitación de los ámbitos extractivos, existía un conflicto entre el sector extractivo y la población cercana, la designación de los mismos es vista desde la población como una dilatación en el tiempo de los problemas existentes. La solución a esta conflictividad pasa ineludiblemente por procesos de participación publica en el desarrollo de los PTP.

\begin{tabular}{|l|c|c|c|}
\hline \multicolumn{1}{|c|}{ Uso } & $\begin{array}{c}\text { Superficie de los ámbitos } \\
\text { extractivos }\left(\mathbf{m}^{3}\right)\end{array}$ & $\begin{array}{c}\text { Necesidades } \\
\text { anuales }\left(\mathbf{m}^{3}\right)\end{array}$ & $\begin{array}{c}\text { Tiempo previsto de } \\
\text { abastecimiento (años) }\end{array}$ \\
\hline $\begin{array}{l}\text { Áridos para hormigones, } \\
\text { aglomerados y morteros }\end{array}$ & 82000000 & 2150000 & 38 \\
\hline Áridos para prefabricados & 49125277 & 840000 & 59 \\
\hline Áridos industriales (puzolana) & 46260000 & 300000 & 154 \\
\hline
\end{tabular}

Tabla 3. Estimación del período de tiempo previsto de suministro de áridos obtenidos en las áreas extractivas autorizadas. El cálculo de las necesidades anuales se basa en el consumo de cemento medio de los años 2005 a 2009 (BOC, 2002; INTERRA; 2011).

Table 3. Estimation of the predicted time period of aggregate supplies from the authorized extractive areas. The calculation of annual requirements is based on average cement consumption of the years 2005-2009(Boc, 2002; INTERRA, 2011). 
Hernández-Durán, G., et al., 2016. Análisis comparativo de propuestas para la... Boletín Geológico y Minero, 127 (4): $755-773$

\section{Propuestas alternativas de ordenación de la actividad extractiva en Tenerife}

Las propuestas alternativas se han generado mediante el empleo de una metodología de ordenación minero-ambiental sustentada en un análisis territorial que incorpora todas aquellas variables que merecen ser consideradas en la selección de las zonas mejores para la implantación de la actividad extractiva. El fundamento metodológico es la valoración de la capacidad de acogida del territorio ante la explotación minera a través de un balance entre la vulnerabilidad o fragilidad ambiental ante la explotación de los recursos geológicos, y la potencialidad que presenta para el desarrollo de dicha actividad (e.g.: Barettino et al., 1994; Hernández-Durán, 2000; Martínez et al., 2006; INTERRA, 2007; Arranz-González y Alberruche del Campo, 2008). La capacidad de acogida puede entenderse como la idoneidad del territorio para sostener una actividad, teniendo en cuenta simultáneamente la medida en que el territorio cubre los requerimientos de localización, así como los efectos de la misma sobre el medio (Gómez Orea, 1994). Normalmente el análisis territorial de la capacidad de acogida se realiza mediante herramientas de información geográfica (SIG), realizando una valoración de los elementos del medio existentes en el territorio o en subdivisiones del mismo. El resultado final son mapas de ordenación minero-ambiental o mapas de compatibilidad minero-ambiental. Considerando los efectos que la minería produce sobre el territorio, y existiendo ya una definición de subunidades establecida en el Plan de Ordenación (BOC, 2011) Ilamadas Áreas de Regulación Homogéneas (ARH en adelante), se decidió valorar la capacidad de acogida de dichas unidades, sin perder de vista las recomendaciones ya establecidas sobre las mismas. También se trabajó con la delimitación de los diferentes Espacios Naturales protegidos y Áreas de Sensibilidad Ecológica, recogidas en el Decreto Legislativo 1/2000 (BOC, 2000) y en las normas que se citan en él. La información de base se obtuvo a través del Cabildo de Tenerife. Los mapas finales que recogen las propuestas de ordenación se realizaron a escala 1:250000.

\section{Análisis del potencial geológico de materiales de construcción en Tenerife}

Sin duda, una integración efectiva de la minería en la ordenación territorial pasa por conocer con suficiente detalle y delimitar geográficamente aquellas zonas del territorio que presentan aptitud para la explotación de los recursos mineros, evaluando la cantidad y calidad de éstos (Barettino et al., 2003). Por ello, además de la aptitud de una determinada litología para un tipo de uso concreto, que depende de sus características físicas (dureza, resistencia, densidad, etc.) y químicas (alterabilidad, reacción con otros componentes, etc.), existen otras variables que influyen directa o indirectamente en su calidad. En el caso de los áridos de construcción que provienen del machaqueo de rocas, y de los picones para prefabricados, es importante el grado de alteración. Cuanto más antiguos y más expuestos a climas húmedos, mayor es la alteración de la roca y menor la calidad. En el caso de los áridos de barranco, la calidad se reduce cuanto mayor sea la cantidad de material fino que tengan (limos y arcillas). Para evaluar estos aspectos se revisó la cartografía geológica de Tenerife y se realizó una clasificación de los recursos geológicos de la isla, según su posible uso y su calidad. La base del trabajo fue el Mapa Geológico Digital Continuo de Canarias y su correspondiente leyenda (Bellido Mulas et al., 2011). Este trabajo está disponible en un formato compatible con SIG, lo que permite un análisis territorial del mismo Las 211 litologías que contiene el mapa se agruparon en función de su aptitud para ser explotadas, teniendo en cuenta para dicha valoración los trabajos ITGE (1973) y Ayala et al. (1986). Para cada uso se evaluó la calidad en base a la edad de los materiales, y a su idoneidad para un uso principal y otro secundario, en función de otras características descriptivas asociadas a cada litología, considerándose suficiente establecer tres categorías de calidad (Fig. 2). Se pensó que convenía realizar el análisis agrupando los recursos según los usos o aprovechamientos principales como áridos: áridos para la construcción (hormigones, aglomerado asfáltico y morteros), áridos para prefabricados, y áridos industriales (puzolanas). Como resultado se obtuvieron una serie de planos de recursos geológicos que son fundamentales para la planificación de la actividad extractiva, pues reconocen las principales zonas de localización de los recursos según tipologías y calidades asociadas, pudiendo servir como herramienta de decisión para priorizar las zonas de extracción.

También es importante la localización de los yacimientos de áridos, ya que al ser recursos de bajo valor, el transporte influye notablemente en el precio de los mismos. Un buen yacimiento de áridos por su calidad y volumen puede ser inviable si no tiene una localización cercana a centros de consumo o vías rápidas. Mediante análisis SIG se puede zonificar el territorio en base a su localización estratégica.

Otro aspecto a tener en cuenta es el volumen del yacimiento, el cual puede ser evaluado reconociendo 


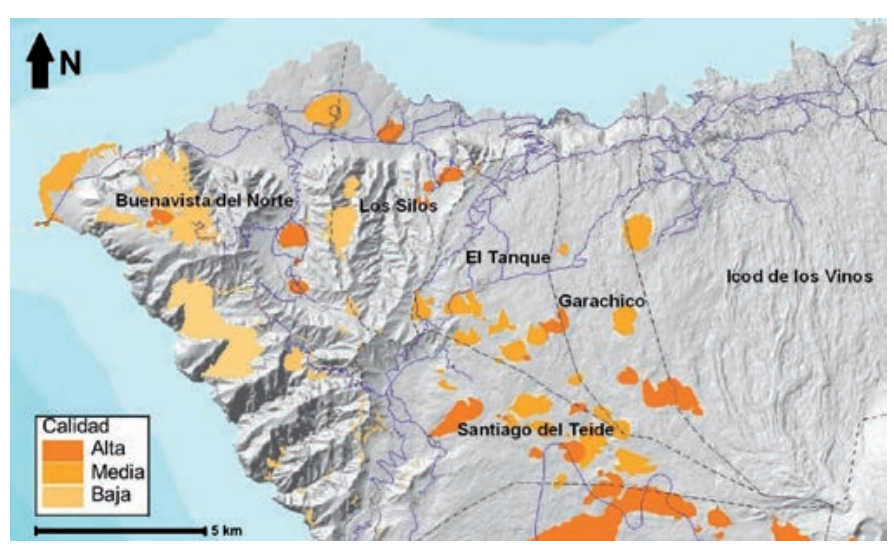

Figura 2. Detalle del plano de localización de recursos geológicos empleados como áridos para prefabricados. Clasificación según la calidad del recurso (modificado de Bellido Mulas et al., 2011).

Figure 2. Detail of the map of geological resources used as aggregates for prefabricated concrete pieces. Classification according to the quality of the resources (adapted from Bellido Mulas et al., 2011).

los espesores de las capas explotables y su extensión en superficie o subsuelo cercano. Sin embargo, su determinación requiere de estudios de detalle que quedaban fuera del alcance de este trabajo. Salvo los casos como el de las gravas de Güímar (donde las excavaciones han revelado su espesor) y el de los conos de picón, resulta imposible cubicar el volumen de los recursos si no se cuenta con estudios de detalle.

\section{Determinación de la capacidad de acogida, criterios de exclusión, variables restrictivas y condicionantes}

Para cualquier modelo de ordenación territorial por el que se opte, se han de establecer unas limitaciones a la implantación de la actividad minera, que permitan preservar los valores ambientales de la isla. Hay que tener en cuenta que la actividad extractiva a cielo abierto genera cambios drásticos en la fisiografía del terreno, y sobre el sistema natural de drenaje. La estructura geológica es también modificada irreversiblemente, aún cuando sea posible reconstruir parcialmente la morfología original del terreno. El ambiente biológico es completamente destruido, o tremendamente transformado, por lo menos mientras se realizan labores mineras (Tandy, 1979). La explotación de áridos implica la eliminación de la vegetación o los usos del suelo, del propio suelo y de la fauna en la superficie afectada, pudiendo añadir además cualquier otro elemento enterrado o situado sobre el suelo dentro de dicha superficie (restos arqueológicos, caminos, etc.), por lo que, desde el punto de vista territorial, la medida del impacto puede ser directamente equiparable al valor del tipo de uso del suelo en riesgo de ser afectado (Arranz-González et al., 2009). A todo esto hay que añadir los efectos temporales de la explotación.

Por todo lo anterior, los modelos propuestos parten de que no se podrá llevar a cabo la actividad extractiva en las siguientes zonas: los Espacios Naturales Protegidos, las Zonas Especiales de Conservación (ZEC), las Zonas de Especial Protección para las Aves (ZEPA), los Bienes de Interés Cultural (BIC, que son mayoritariamente arqueológicos). A estos espacios, se sumaron los cauces de barrancos, considerados Áreas de Protección Ambiental 1, y las playas, que estarían incluidas dentro de las llamadas Áreas de Protección Ambiental 3 (BOC, 2011). Todas estas zonas, excluidas desde un primer momento, se pueden considerar una restricción impuesta por la normativa vigente, aunque no cabe duda de que un análisis independiente reconocería una muy baja o nula capacidad de acogida para la actividad extractiva.

Desde el punto de vista normativo existe también una evidente conflictividad de usos entre zonas urbanas y explotación de recursos mineros, a lo que se suman los efectos producidos por la actividad minera (ruido, vibraciones, emisión de partículas de polvo, tráfico pesado, etc.), que son los que afectan en mayor medida a la población. Por ello, se consideró justificada la exclusión de estas áreas, incluyendo las llamadas ARH Urbanas, las ARH de Expansión Urbanas, y las ARH de Interés Estratégico (IE) (BOC, 2011): que son los ámbitos que ostentan un papel estratégico en el Modelo de Ordenación Territorial, por estar destinados a albergar equipamientos o infraestructuras de servicio insular.

Siguiendo la terminología empleada en IGME (1995) todas las anteriores zonas poseerían una capacidad de acogida excluyente, derivada de un elevado impacto, independientemente de la aptitud geológico-minera. En ellas la localización de explotaciones se consideró inaceptable, por lo que la actividad extractiva debe estar prohibida. El resto del territorio fue considerado Zona de Estudio Potencial para el desarrollo de la actividad extractiva en un análisis posterior.

Para establecer nuevos niveles de capacidad de acogida de cara a la explotación de recursos de áridos, fueron considerados otra serie de aspectos que se denominaron variables restrictivas. No cabe duda de que sin llegar a ser merecedoras de la total exclusión, determinadas zonas presentan un alto grado de incompatibilidad con la minería. En primer lugar están las zonas de alta calidad forestal, incluyendo en este grupo las denominadas ARH de Protección Ambiental 2: Bosques consolidados y Bosques 
Hernández-Durán, G., et al., 2016. Análisis comparativo de propuestas para la... Boletín Geológico y Minero, 127 (4): $755-773$

potenciales, son aquéllas zonas que, en virtud de sus características físicas y ecológicas, son o están llamadas a ser áreas de bosque. Estas áreas presentan un alto interés natural y deben cumplir el triple papel de ser elementos relevantes del paisaje, mantenedores de procesos ecológicos esenciales y soporte de gran parte de los usos recreativos vinculados al medio natural, por lo que deben ser espacios de especial protección. También se consideró que deberían someterse a niveles semejantes de restricción ante la actividad extractiva los terrenos incluidos dentro de franjas de protección de $500 \mathrm{~m}$, medidos desde los límites de las zonas de protección (ENP, ZEC y ZEPA), buscando una amortiguación frente a los impactos derivados del ruido, así como de las partículas de polvo más pesadas que caerían directamente sobre la vegetación. De modo semejante se establecieron franjas de protección de $500 \mathrm{~m}$ entre el sector litoral y el interior, teniendo en cuenta que la normativa de ordenación vigente considera incompatible la actividad minera en zonas costeras. También se establecieron franjas de protección de $500 \mathrm{~m}$ de cara a limitar la posible afección a poblaciones, dado que la afección directa a la población es una de las mayores fuentes de conflicto, originadas por la merma de las condiciones de salud y sosiego público derivados del ruido, polvo, vibraciones y tráfico pesado. La fijación de un radio de $500 \mathrm{~m}$ respecto a los límites de las llamadas Áreas de regulación Homogénea Urbanas y de Expansión Urbanas, obedece a que a esa distancia los niveles sonoros de una explotación pueden reducirse en más del $50 \%$, es la franja que recibe las partículas de polvo más pesadas, y concentra la mayoría del tráfico. Por último se estableció una franja de protección alrededor de los bienes de interés cultural (BIC) declarados de $100 \mathrm{~m}$ respecto a sus límites externos, para prevenir una afección a estos bienes por el deslizamiento o desplome de taludes, y amortiguar las vibraciones producidas por el uso de martillos o voladuras.

En todas las zonas en las que se cumplen los criterios anteriormente expuestos, ligados a las variables restrictivas, se consideró que la explotación de recursos mineros deberá prohibirse salvo en casos de excepción, y estar vinculada únicamente a obras de interés insular, o situaciones especiales en las que el aprovechamiento de un determinado recurso sea únicamente viable en una ubicación concreta. De este modo se incorporó el espíritu de la normativa vigente de ordenación. Siguiendo la terminología empleada en IGME (1995) se trataría de zonas con una capacidad de acogida baja, en las que los impactos pueden ser elevados y la localización de explotaciones se considera compatible con limitaciones, marcadas estas últimas por la existencia de unas condiciones de aptitud geológico-minera muy específicas.

Además de todo lo anterior, en el diseño de las diferentes propuestas de ordenación se consideró que existía un grado de incompatibilidad mayor en aquellos fragmentos del territorio en los están presentes las siguientes variables condicionantes: los terrenos incluidos en áreas de importancia para las aves (International Bird Areas, IBAs), los terrenos agrícolas de las ARH de Protección Económica 1 y 2, los Ámbitos de Referencia Turísticos, las zonas cercanas a zonas urbanas, las Áreas de Sensibilidad Ecológica (BOC, 2000).

Si bien las IBAs carecen del rango normativo otorgado a otras figuras de protección, se consideró aconsejable condicionar el desarrollo de actividades mineras, dado que éstas pueden provocar la destrucción de hábitats, la destrucción de nidos, y generar perturbaciones en el comportamiento de las aves (apareamiento, nidificación, migraciones, etc.) a causa del ruido, polvo, vibraciones, etc. Igualmente, no cabe duda de que la actividad extractiva puede suponer la destrucción de valores geológicos y geomorfológicos. Las principales áreas y enclaves de interés geológico-geomorfológico ya fueron previamente descartadas del análisis al coincidir con las diferentes figuras de protección que integran la Red Canaria de ENP. En la normativa de ordenación vigente, las denominadas ARH de Protección Ambiental 1 son espacios de alto interés geomorfológico, requiriendo de la preservación de su estructura física, geomorfológica y paisajística, así como de los ecosistemas asociados. Estas ARH comprenden, al margen de los barrancos, que ya han sido considerados más arriba: laderas, malpaíses y llanos, y montañas. Igualmente puede ocurrir en las Áreas de Sensibilidad Ecológica. Por otro lado, el importante papel que el desarrollo de la actividad agrícola ha tenido como creadora y sustentadora del paisaje de la isla aconseja incorporar como variables de análisis aquellos espacios considerados como ARH de Protección Económica 1 y 2. Se incluyen en esta categoría los terrenos que reúnen condiciones ambientales más favorables para el desarrollo de la actividad agrícola, atendiendo a su potencial productivo. También se consideraron los denominados Ámbitos de Referencia Turísticos (BOC, 2011), en los que puede existir un evidente conflicto con las explotaciones mineras. Por último, y aunque ya ha sido considerada, la existencia de áreas urbanas a cierta distancia puede ser motivo para limitar la explotación de áridos. Con esta idea, se consideró que, aun permitiéndose la actividad en las zonas que distan al menos $500 \mathrm{~m}$ de zonas urbanas y de expansión urbanística, la implantación de la actividad debe 
Hernández-Durán, G., et al., 2016. Análisis comparativo de propuestas para la... Boletín Geológico y Minero, 127 (4): $755-773$

estar supeditada a la adopción de limitaciones o medidas de mitigación de impactos cuanto mayor cercanía exista a los núcleos poblados. Se propuso crear otra franja mínima de $1000 \mathrm{~m}$ respecto a las zonas urbanas donde habrían de aplicarse dichas medidas de mitigación. La fijación de este umbral de $1000 \mathrm{~m}$ responde a un límite a partir del cual la mitigación de ruido es tan acusada que resulta difícil diferenciar el ruido originado por la actividad minera del ruido ambiente. Para el resto de factores como el polvo y el tráfico, a partir de este umbral se empieza a perder la relación lineal entre distancia y atenuación de impactos, al intervenir variables climáticas y de red viaria.

Con un grado de incompatibilidad menor, se estimó que las áreas con determinado grado de pendiente $\left(<20,20-40,>40^{\circ}\right)$, la actividad sólo debería implantarse bajo determinadas condiciones. Esta variable no condiciona notablemente la implantación de la actividad extractiva, si bien es notorio que dicha actividad resulta más impactante cuando se sitúa en zonas de fuerte pendiente, ya que por lo general resulta más visible. Por ello, se realizó una división del territorio en base a un Modelo Digital del Terreno, con una resolución de malla de $10 \times 10 \mathrm{~m}$, agrupando las pendientes en diferentes intervalos que requerirán la adopción de medidas complementarias de mitigación del impacto paisajístico.

Además de los aspectos anteriores, han de considerarse también aquellos que incrementan la compatibilidad, destacando la proximidad a explotaciones mineras activas, abandonadas y no restauradas. A tal fin, se estableció un área de influencia de 500 $\mathrm{m}$ alrededor de las extracciones, donde en igualdad de condiciones, se implantaría preferentemente la actividad. Esta distancia alrededor de las explotaciones existentes, donde existe una degradación del territorio, se justifica con argumentos similares a los empleados para prohibir las extracciones situadas a menos de $500 \mathrm{~m}$ de zonas naturales de alto valor y zonas pobladas.

Todas las consideraciones anteriores permiten identificar zonas en las que, siguiendo la terminología empleada en IGME (1995), la capacidad de acogida sería media, en las que la localización de explotaciones se considera compatible con limitaciones marcadas por un nivel de exigencia mayor en el desarrollo de las prácticas propias de la actividad. En todo el resto del territorio insular, a la escala de trabajo empleada, se puede afirmar que la capacidad de acogida es alta, con niveles aceptables de impacto, considerando la posterior rehabilitación del terreno, por lo que el uso extractivo puede considerarse vocacional donde existan recursos de calidad. Esta sistemática admitiría la inclusión de nuevos condicionantes de aplicarse a una escala más detallada, en la que pueden hacerse visibles efectos o conflictos de importancia local, siendo válido todo lo anteriormente dicho sobre criterios y variables excluyentes o condicionantes. Los resultados que arroja la aplicación de esta metodología se asemejarían, con mayor refinamiento y nivel de exigencia, a la aplicación de los métodos para la delimitación de áreas de salvaguarda (safeguarding areas), como los empleados en Gran Bretaña (McEvoy et al., 2007; Wrighton y Humpage, 2012).

\section{Resultados}

\section{Aplicación de los criterios y variables excluyentes y condicionantes a la actividad extractiva de áridos en Tenerife: modelo zonificado}

Para el establecimiento de las propuestas de ordenación en Tenerife, se partió de la aptitud geológico-minera, contando únicamente con las áreas que fueron cartografiadas dentro de las categorías de calidad media y alta para los diferentes tipos de uso o aprovechamiento principal: áridos para la construcción, áridos para prefabricados, y áridos industriales. En la Fig. 3 se esquematiza la metodología seguida.

Como se explica más arriba, el punto de partida es la división territorial en Zonas de Protección y Zonas de Estudio Potenciales. El segundo paso consiste en realizar una clasificación de las Zonas de Estudio Potenciales a partir del análisis territorial de la presencia de las variables restrictivas y condicionantes comentadas en el epígrafe anterior. Se consideró que las variables restrictivas tendrían prioridad frente a las condicionantes, en el caso de coincidir ambas territorialmente. El tercer paso consistió en determinar qué parte del territorio ha sido afectada por la actividad extractiva previa, derivada de la existencia de extracciones activas y abandonadas sin restaurar. El último paso consistió en la reclasificación de las zonas identificadas en función de si estaban o no afectadas previamente por minería. Esta reclasificación solo afectó a las zonas de mayor capacidad de acogida: ámbitos de referencia turísticos, Áreas de Regulación Homogéneas de Protección Económica 1 y 2 (terrenos que reúnen condiciones ambientales para el desarrollo de la actividad agrícola), y resto del territorio. Según el que se denominó modelo zonificado, el territorio es clasificado en cinco nuevas categorías, en las que la actividad debería tener distinto grado de implantación en función de su capacidad de acogida. Éstas son:

- Zonas de actividad extractiva prohibida que incluye las zonas de protección reconocidas inicialmente 
y las zonas sin recursos o con recursos de baja calidad.

- Zonas de actividad extractiva prohibida salvo casos de excepción, definidas en base al reconocimiento de alguna de las variables restrictivas descritas más arriba (las de menor capacidad de acogida para la actividad extractiva). Los casos de excepción deben estar vinculados únicamente a obras

\section{MODELO ZONIFICADO}

Zonas de protección

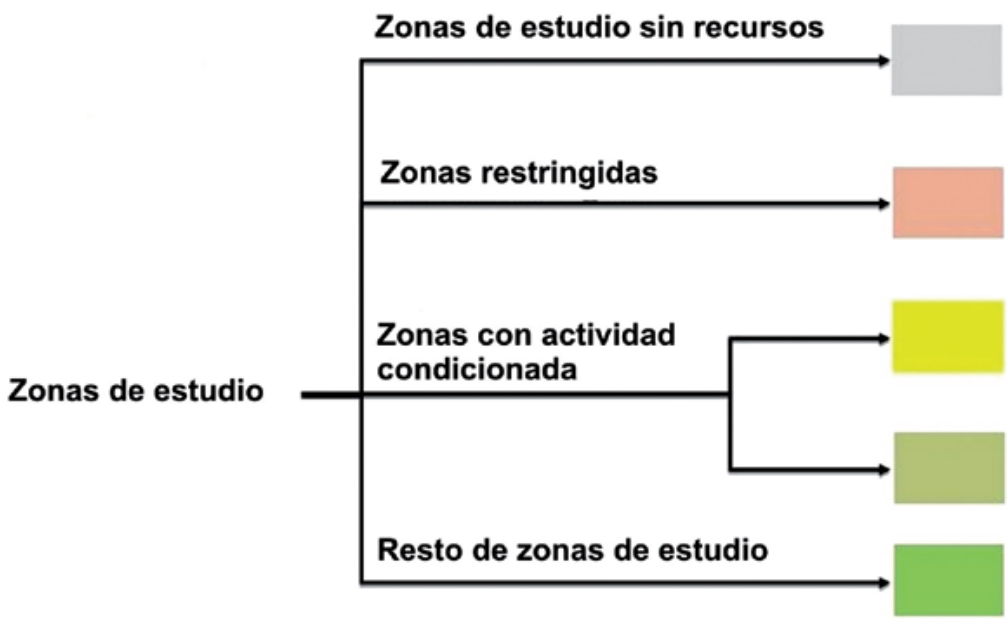

ACTIVIDAD EXTRACTIVA PROHIBIDA

ACTIVIDAD EXTRACTIVA DESCARTADA

ACTIVIDAD EXTRACTIVA PROHIBIDA SALVO CASOS DE EXCEPCIÓN

ACTIVIDAD EXTRACTIVA CONDICIONADA. LIMITACIÓN DE LAACTIVIDAD+ CONDICIONANTES TÉCNICOS

ACTIVIDAD EXTRACTIVA CONDICIONADA. CONDICIONANTES TÉCNICOS

ACTIVIDAD EXTRACTIVA PERMITIDA SIN CONDICIONANTES
1.-PUNTO DE PARTIDA: ZONAS DE ESTUDIO A ESCALA INSULAR
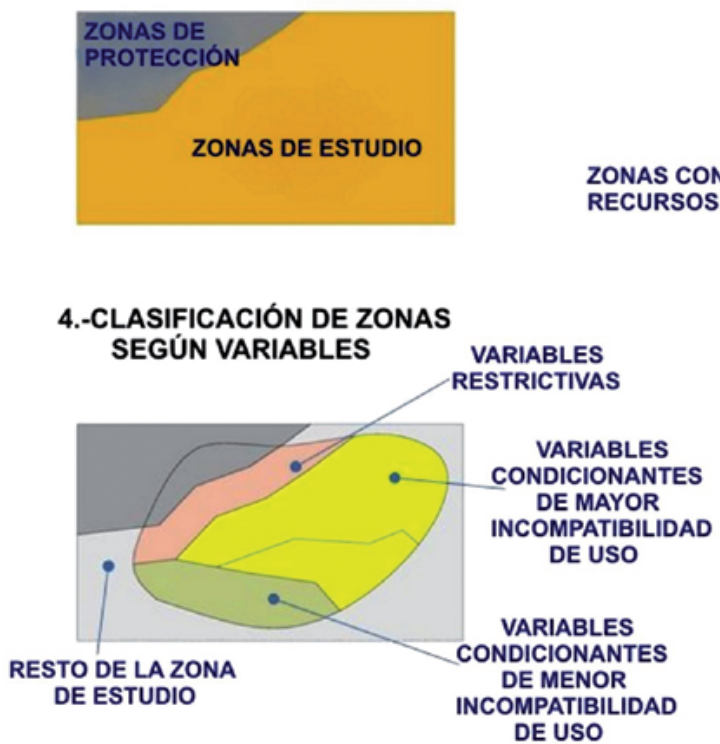

2.-IDENTIFICACIÓN DE ZONAS CON RECURSOS

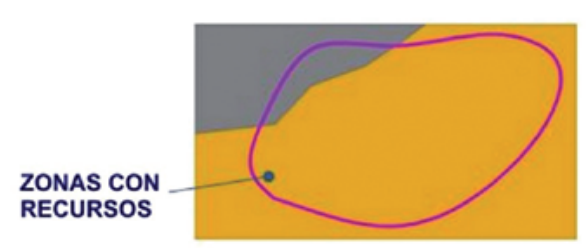

5.-DETERMINACIÓN DE LAS ZONAS AFECTADAS POR LAS EXTRACCIONES

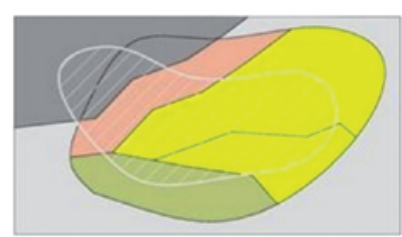

3.-DELIMITACIÓN DE ZONAS DE ESTUDIO CON RECURSOS

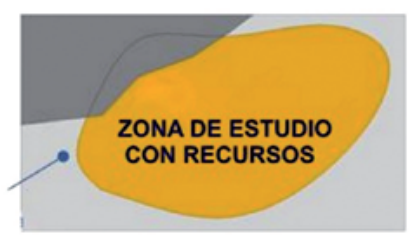

\section{6.-RECLASIFICACIÓN DE POLIGGONOS Y RESULTADO FINAL}

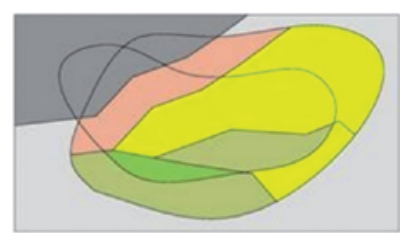

Figura 3. A: Esquema de las categorías de ordenación territorial propuestas en el modelo zonificado. B: Diagrama esquemático del procedimiento seguido para obtener el modelo de planificación zonificado.

Figure 3. A: Scheme of the proposed land planning zoning categories of the zoned planning model. B: Schematic diagram of the procedure to obtain the zoned planning model. 
Hernández-Durán, G., et al., 2016. Análisis comparativo de propuestas para la... Boletín Geológico y Minero, 127 (4): $755-773$

de interés insular, o situaciones en las que el aprovechamiento de un recurso sea únicamente viable en una cierta ubicación.

- Zonas de actividad extractiva limitada y condicionada, definidas en base a que en ellas se identificó alguna de las variables condicionantes de mayor grado de incompatibilidad. En estas zonas la explotación puede estar permitida, pero su implantación conllevaría la aplicación de ciertas limitaciones de la actividad (temporales, de producción anual, de superficie de ocupación, y de volumen, y profundidad de la excavación), y además una serie de condicionantes técnicos encaminados a disminuir los impactos (tipología y desarrollo de los huecos de explotación, vertidos de estériles, ubicación de instalaciones, exigencias especiales de restauración del terreno, medidas de reducción del impacto visual y uso de explosivos).

- Zonas de actividad extractiva condicionada, en función del grado de pendiente, donde se consideró necesario contemplar una serie de condicionantes técnicos semejantes a los de las zonas anteriores, pero sin limitaciones de la actividad.

- El resto del territorio albergaría las zonas de actividad extractiva permitida sin condicionantes. Territorialmente coincide con las áreas con recursos aptos donde no existen ni variables restrictivas ni condicionantes, estando además influenciadas por actividad extractiva previa. En estas zonas la actividad se podría desarrollar sin ningún tipo de condicionantes, salvo los que impone la legislación sobre minas y seguridad minera, la cual es de aplicación en cualquier caso.

La aplicación de estos criterios sobre el territorio tinerfeño da lugar a los mapas de ordenación para cada uno de los tres tipos de recurso según el modelo zonificado.

La Figura 4 muestra el mapa resultante relativo a los recursos aptos como áridos para hormigones, aglomerados y morteros. Se incluyeron, como se ha dicho, las zonas con recursos de calidad media y alta, añadiendo también la vertiente SE de la isla donde están presentes superficialmente depósitos de pumitas. Esta consideración se apoya en que en algunos casos la cobertura de pumitas no impide la explotación de rocas infrayacentes aptas para estos usos. En cuanto a la influencia de la actividad extractiva previa se tuvieron en cuenta las canteras inventariadas de gravas, arenas y roca (fonolita o basalto), que se encontraban activas o abandonadas sin restaurar. El resultado es que la actividad resultaría prohibida en un $63 \%$ del territorio por tratarse de zonas de protección $y$ en un $4 \%$ por inexistencia de recurso (un total de
137124 ha). La actividad estaría prohibida salvo casos de excepción en un $20 \%$ del territorio (40994 ha). En el $12 \%$ del territorio donde se permite la actividad, las categorías se distribuyen superficialmente de la siguiente manera: un $11 \%$ del territorio podría albergar explotaciones con limitaciones y condicionantes técnicos ( 21623 ha); un $1.60 \%$ podría albergar explotaciones con condicionantes técnicos, (3248 ha), y un $0.16 \%$ del territorio podría albergar explotaciones sin ningún tipo de condicionantes (328 ha). La aplicación de este modelo zonificado llevaría previsiblemente a la concentración de las explotaciones en aquellos lugares donde no existen limitaciones o condicionantes para su implantación. A pesar de que en un alto porcentaje de la superficie, la actividad estaría limitada o condicionada, el terreno disponible sería con creces muy superior al necesario. La distribución cartográfica de las distintas zonas que se derivan de este modelo permite observar que existiría un buen equilibrio comarcal. Si se calculan las necesidades productivas en $2150000 \mathrm{~m}^{3}$ anuales, con tan solo una profundidad de excavación neta (de producto explotable) de $25 \mathrm{~m}$, y aunque se limitara la actividad a las 328 ha de mayor capacidad de acogida, las necesidades estarían cubiertas durante casi cuarenta años.

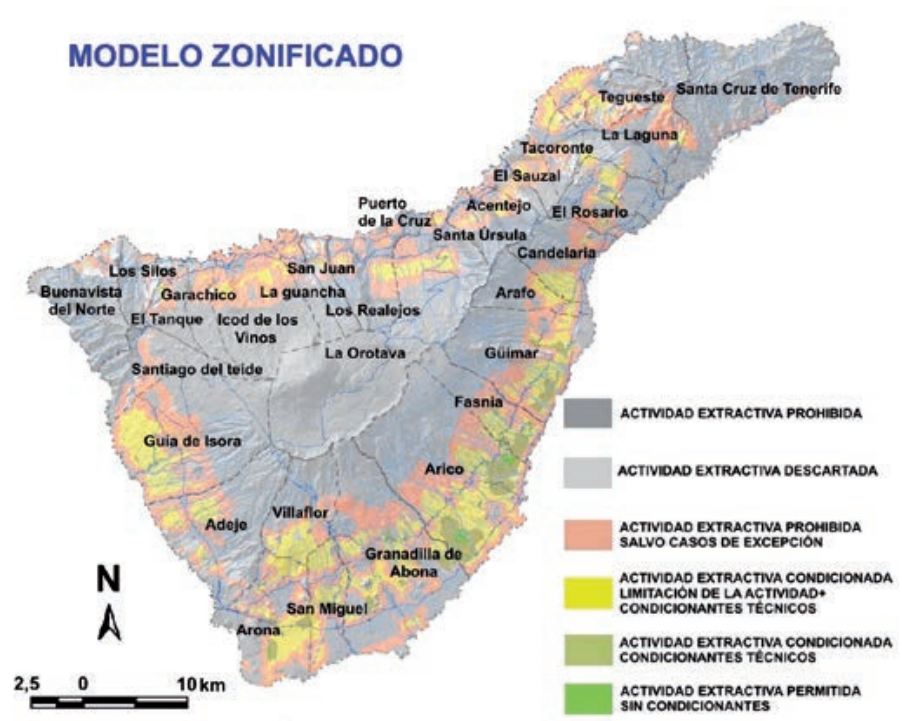

Figura 4. Mapa que muestra las diferentes categorías propuestas en las que la actividad debe tener un diferente grado de implementación (referido a áridos para la construcción), como resultado de la aplicación del modelo de planificación zonificado (modificado de INTERRA, 2011).

Figure 4. Map showing the proposed different categories in which the activity should have a different degree of implementation (referring to aggregates for construction), as a result of the application of the zone- planning model (adapted from INTERRA, 2011). 
En el modelo zonificado para los áridos de prefabricados se consideraron todas las variables restrictivas y condicionantes expuestas más arriba, salvo uno de los aspectos del valor geológico-geomorfológico. De las tres subcategorías que comprenden las Áreas de Regulación Homogénea de Protección Ambiental 1 (laderas, malpaíses y llanos, y montañas), no se consideraron las montañas, puesto que salvo los conos volcánicos reconocidos actualmente como ámbitos extractivos, el resto pertenecen a esta categoría. Sin esa condición no sería posible evaluar distintas opciones de ordenación diferentes a la actualmente vigente. A la hora de seleccionar los recursos aptos para estos usos se tuvieron en cuenta las zonas con recursos de calidad media y alta en el correspondiente mapa de calidades de recursos aptos como áridos para prefabricados. Ya se mencionó que los recursos empleados como áridos para prefabricados son los denominados localmente como picones, que son piroclastos de tamaño lapilli, generalmente basálticos, y que presentan un bajo grado de alteración. Se localizan alrededor de centros eruptivos (conos volcánicos), concentrados mayoritariamente en torno a las Ilamadas dorsales de la isla (NE, S y NW), siguiendo los trabajos de Carracedo $(1994,2008)$. Los conos volcánicos de la dorsal NW están próximos al centro de la isla, que coincide aproximadamente con las principales zonas protegidas, y están prácticamente ausentes en la zona norte. En cuanto a la influencia de la actividad extractiva previa se consideraron las canteras de picón inventariadas que se encontraban activas o abandonadas sin restaurar. Los recursos aptos para áridos de prefabricados que se encuentran dentro de la zona de estudio ocupan tan solo un $1 \%$ del territorio insular. Pero además, dentro de ese 1\%, más de la mitad de la superficie (55\%) coincide con zonas donde están presentes variables restrictivas 0 condicionantes (3004 ha), con lo que la actividad estaría totalmente permitida según el modelo solo en algunos de los conos (209 ha). La distribución de las zonas donde se permitiría la actividad no está equilibrada territorialmente. Esta circunstancia es debida no solo a la cercanía a poblaciones en la zona NE, sino que en parte es debida a la distribución propia del recurso. Al permitirse la extracción no solo en aquellos conos volcánicos de mayor aptitud, sino en los de aptitud intermedia siempre que se apliquen limitaciones y condicionantes, supone una buena garantía de suministro. No obstante, si se calculan las necesidades productivas en $840000 \mathrm{~m}^{3}$ anuales, con tan solo una profundidad de excavación neta (de producto explotable) de $25 \mathrm{~m}$, y aunque se limitara la actividad a las 209 ha de mayor capacidad de acogida, las necesidades estarían cubiertas durante más de sesenta años.
Para el análisis territorial referente a los recursos aptos como áridos industriales (puzolanas) se incluyeron igualmente las zonas con recursos de calidad media y alta, y las canteras de jabre o puzolana activas o abandonadas sin restaurar. El recurso se localiza mayoritariamente en la vertiente SE de la isla, desde el valle de Güímar hasta Granadilla, con pequeños afloramientos en la zona metropolitana y el Valle de La Orotava. El modelo reconoce una superficie de un $6 \%$ del territorio insular en el que existen recursos y la actividad no estaría completamente descartada. Considerando únicamente la superficie con recursos, un $46 \%$ ( 5715 ha) correspondería a actividad prohibida salvo excepción; un 39\% (4866 ha) correspondería a zonas con limitaciones y condicionantes técnicos; un $12 \%$ (1455 ha) tendría condicionantes técnicos, y la actividad se permitiría sin condicionantes en un $3 \%$ (442 ha). Este tipo de recursos, al tener una localización bastante concreta no genera una excesiva dispersión de la actividad, y por ello, la distribución de las zonas donde se permitiría la actividad no está equilibrada territorialmente. Sin embargo, estos recursos se consumen directamente en fábrica (localizada en Santa Cruz) o se exportan en barco, por lo que la distribución equilibrada no es tan importante como en los otros tipos de áridos. El modelo es suficientemente capaz de cubrir una demanda anual calculada de $300000 \mathrm{~m}^{3}$ durante más de 350 años para profundidades de excavación de $25 \mathrm{~m}$, ya que la zona donde se permitiría la actividad sin limitaciones es de 442 ha. Pese a que desde el punto de vista teórico el modelo tiene un buen comportamiento, es necesario mencionar que existe la posibilidad de que una determinada explotación se encuentre en diferentes zonas del modelo, lo que puede complicar la aplicación de las limitaciones y los condicionantes previstos para cada zona.

\section{Aplicación de los criterios y variables excluyentes y condicionantes a la actividad extractiva de áridos en Tenerife: modelo zonificado-concentrado}

Como se ha mencionado se trabajó también en la elaboración de propuestas de ordenación para los tres tipos de recursos siguiendo lo que se denominó modelo zonificado-concentrado. Como puede verse en los esquemas de la Fig. 5, el punto de partida es el modelo zonificado para cada uno de los tres tipos de recursos. Se eligieron las zonas que representan las dos clases de mayor capacidad de acogida después de la reclasificación posterior a la incorporación de la existencia de áreas ya explotadas. Es decir, solamente se incluyeron aquellas zonas donde no se 
reconocieron ni variables restrictivas ni condicionantes, y además estaban influenciadas por actividad extractiva previa. Además, se consideraron sólo aqueIlas áreas con una superficie suficientemente amplia para constituir un ámbito extractivo al estilo de los propuestos por la normativa vigente. Para ello, se estableció como criterio que los nuevos ámbitos tuvieran una superficie suficiente para albergar al menos dos unidades productivas de tamaño medio. La superficie media de las explotaciones (canteras) que existen en la actualidad en Tenerife se sitúa en torno a las 10 ha, y por tanto en la parte final del análisis territorial se eliminaron los recintos con una superficie inferior a las 20 ha, para permitir un razonable desarrollo de la actividad. En estas zonas la actividad se podría desarrollar sin ningún tipo de condicionantes salvo los que impone la normativa sectorial y ambiental, denominándose zonas de actividad extractiva permitida sin condicionantes. El resto del territorio se calificó como zonas de actividad extractiva prohibida. Como ocurre con el modelo vigente, el territorio resultó así dividido sólo en dos categorías, pero después de un examen más sistemático de la distribución de recursos mineros, además de otras consideraciones ambientales que permiten obtener un resultado bastante conservador, aunque menos que en el modelo zonificado en cuanto a condicionantes de la explotación. En la Tabla 4 se muestran los ámbitos extractivos propuestos para los diferentes tipos de recursos.

Hay que señalar una diferencia con respecto al modelo zonificado en el caso de los áridos para hormigones, aglomerados y morteros. Se buscó que las zonas donde se puedan localizar las explotaciones alberguen recursos en cantidad y calidad suficientes, para obtener un limitado número de ámbitos extractivos, a semejanza del modelo vigente. Por ello, no se incorporaron en esta alternativa las zonas de pumitas de la vertiente SE de la isla. EI resultado obtenido en lo que se refiere a este tipo de recursos de áridos son 7 zonas (Fig. 6) donde la explotación de los mismos estaría permitida. Esto puede suponer una buena garantía de suministro en el caso de bloqueo de alguno de ellos. La actividad extractiva estaría limitada a un $0.40 \%$ de la isla. La capacidad productiva sería suficiente para un periodo de tiempo importante (Tabla 4). Incluso en el caso de eliminar el denominado AEP-1 (situado en los barrancos de Güímar, donde los conflictos que la extracción genera son muy importantes), el modelo permitiría abastecer de áridos a la isla durante 76 años (calculando profundidades de excavación de recurso medias de $25 \mathrm{~m}$ ). Hay que resaltar que la posibilidad de un cese definitivo de la explotación en los barrancos de Güímar es muy elevada. Por otro lado, aunque algunos ámbitos resultan ser de pequeña superficie, permitiendo únicamente la implantación de dos canteras tipo, la mayoría de ellos tienen una superficie superior a las 100 ha. La mayoría de los ámbitos se localizarían en la vertiente SE de la isla. La vertiente norte no tendría ninguna zona y la SW solo una. Esto evidentemente viene condicionado por la geología y una importante carga de proteccionismo.

\begin{tabular}{|c|c|c|c|c|c|}
\hline \multicolumn{2}{|c|}{$\begin{array}{l}\text { Áridos para hormigones, } \\
\text { aglomerados y morteros }\end{array}$} & \multicolumn{2}{|c|}{ Áridos para prefabricados } & \multicolumn{2}{|c|}{ Áridos industriales (puzolanas) } \\
\hline $\begin{array}{c}\text { Ámbito extractivo } \\
\text { propuesto (AEP) }\end{array}$ & Área (ha) & $\begin{array}{l}\text { Ámbito extractivo } \\
\text { propuesto (AEP) }\end{array}$ & Área (ha) & $\begin{array}{c}\text { Ámbito extractivo } \\
\text { propuesto (AEP) }\end{array}$ & Área (ha) \\
\hline AEP-1 & 154 & AEP-1 & 22 & AEP-1 & 182 \\
\hline AEP-2 & 86 & AEP-2 & 44 & AEP-2 & 571 \\
\hline AEP-3 & 144 & AEP-3 & 21 & AEP-3 & 181 \\
\hline AEP-4 & 104 & AEP-4 & 20 & AEP-4 & 85 \\
\hline AEP-5 & 137 & AEP-5 & 31 & AEP-5 & 60 \\
\hline AEP-6 & 160 & AEP-6 & 33 & & \\
\hline AEP-7 & 21 & AEP-7 & 25 & & \\
\hline Total & 806 & Total & 198 & Total & 1.078 \\
\hline
\end{tabular}

Tabla 4. Superficies ocupadas por los ámbitos extractivos propuestos como resultado de la aplicación del modelo de planificación territorial zonificado-concentrado

Table 4. Areas occupied by the proposed extractive zones as a result of the application of the zone-concentrated land planning model. 


\section{MODELO ZONIFICADO-CONCENTRADO}

\section{Zonas de protección}

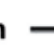

ACTIVIDAD EXTRACTIVA PROHIBIDA

$$
\text { ACTIVIDAD EXTRACTIVA PERMITIDA }
$$
SIN CONDICIONANTES

\section{Zonas de estudio}

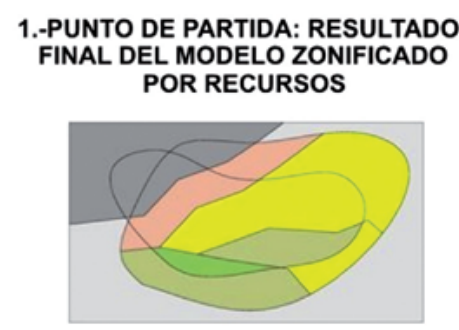

\section{2.-SELECCIÓN DE LAS ZONAS DE MAYOR CAPACIDAD DE ACOGIDA}

\section{ACTIVIDAD EXTRACTIVA PROHIBIDA}

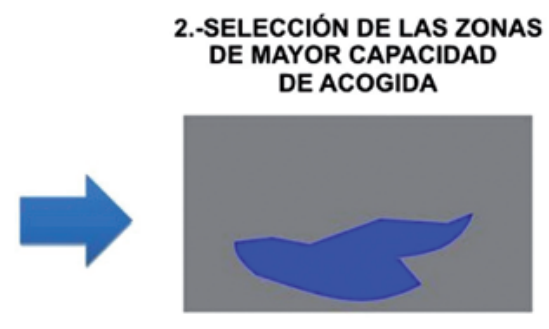

Figura 5. A: Esquema de las categorías de ordenación territorial propuestas en el modelo zonificado-concentrado. B: Diagrama esquemático del procedimiento seguido para obtener el modelo de planificación zonificado-concentrado.

Figure 5. A: Scheme of the proposed land planning zoning categories of the zone-concentrated planning model. B: Schematic diagram of the procedure to obtain the zone-concentrated planning model.

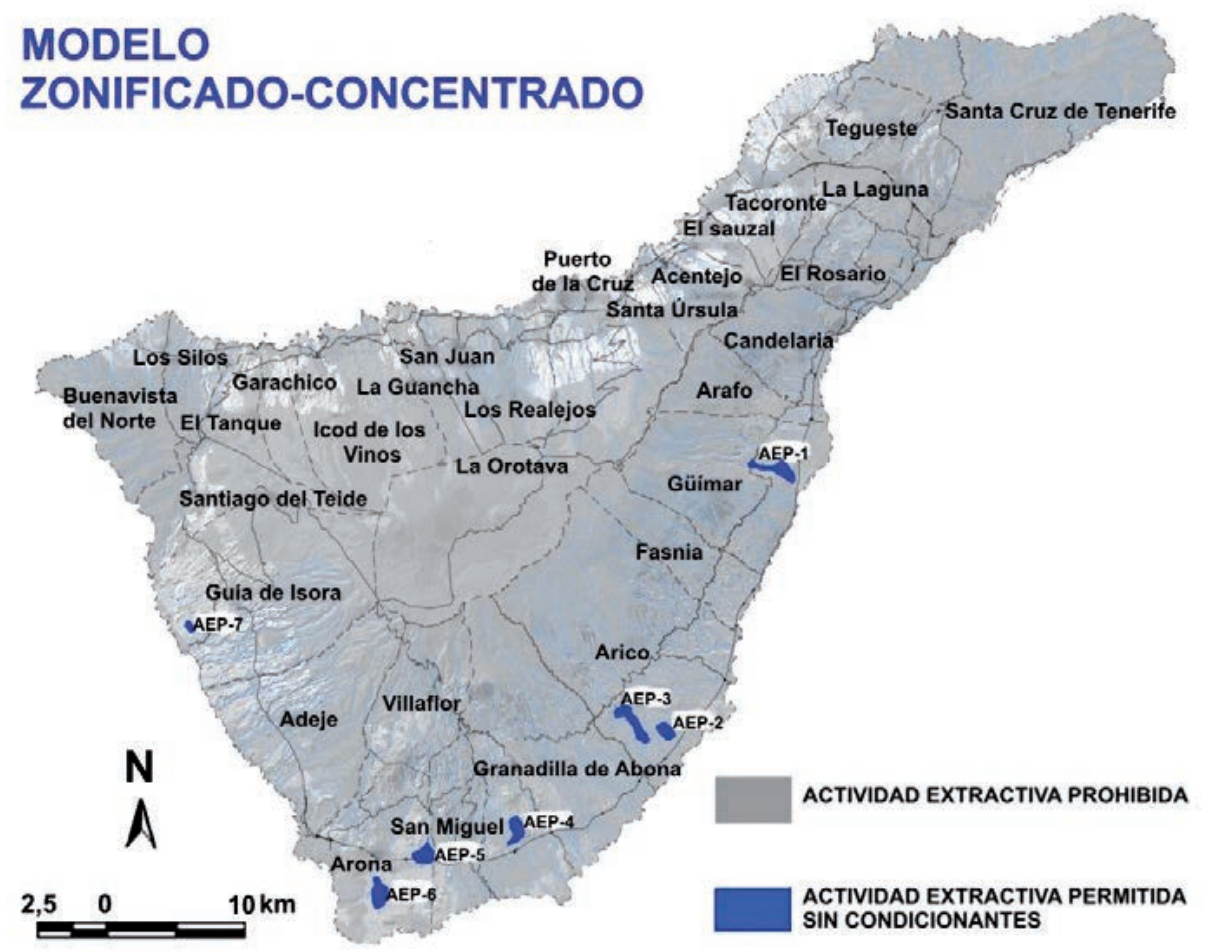

\section{MODELO} ZONIFICADO-CONCENTRADO
Figura 6. Mapa que muestra la localización de las zonas propuestas para la actividad extractiva de áridos para la construcción (azul), como resultado de la aplicación del modelo de planificación zonificado-concentrado (modificado de INTERRA, 2011). El resto del territorio (gris) puede considerarse prohibido para la actividad extractiva.

Figure 6. Map showing the location of proposed extractive zones of aggregates for construction (blue), as a result of the application of the zone-concentrated land planning model (adapted from INTERRA, 2011). The rest of the territory (grey) can be considered as prohibited areas for extractive activities. 
En relación con los áridos para prefabricados, resultaron también 7 zonas donde la actividad extractiva estaría permitida. La mayoría de los ámbitos resultantes es de pequeña superficie, lo que permitiría únicamente la implantación de dos canteras tipo. La mayoría de ellos se localizaría en las zonas SE y $\mathrm{S}$ de la isla, estando otra localizada en la zona NE. La vertiente $\mathrm{N}$ no tendría ninguna zona. La ocupación de suelo de este modelo supone un $0,10 \%$ del territorio insular. La distribución de los ámbitos no estaría equilibrada territorialmente, lo que se debe principalmente a la propia distribución de este tipo de recurso. El modelo permitiría abastecer de áridos a la isla durante 59 años (calculando profundidades de excavación de recurso medias de $25 \mathrm{~m}$ ), resultado prácticamente idéntico al modelo vigente en este aspecto.

En cuanto a los áridos industriales (puzolanas), resultaron 5 zonas donde la explotación de estos recursos estaría permitida. Todas ellas, como es lógico, se localizan en la vertiente SE de la isla. La ocupación de suelo de este modelo sería del $0,53 \%$ del territorio insular. En cuanto al número de ámbitos y a la superficie ocupada, son superiores a las necesarias para un recurso que no tiene una excesiva demanda en la actualidad. Si se calcula una profundidad de excavación neta (de producto explotable) de $25 \mathrm{~m}$, la superficie obtenida permitiría un abastecimiento para casi 900 años. La distribución de los ámbitos no resultó equilibrada territorialmente, debido a la propia distribución del recurso, aunque la distribución equilibrada no es tan importante como en los otros tipos de áridos, como ya se ha comentado.

Teóricamente el modelo zonificado-concentrado presenta las mismas ventajas que el modelo vigente, al posibilitar la coordinación de los proyectos de explotación y restauración en cada uno de los ámbitos, facilitándose el control desde la administración, siempre y cuando se supediten las autorizaciones a la obligatoriedad de lograr esa coordinación entre los responsables de las diferentes explotaciones. Igualmente se ven facilitados los procesos de participación y consulta. En este modelo, por el contrario, todo el potencial geológico conocido ha sido considerado. La metodología seguida para definir los nuevos ámbitos extractivos propuestos en el llamado modelo zonificado-concentrado lleva a que los principales conflictos de usos que pueden surgir en las áreas delimitadas sean con terrenos dedicados a la agricultura

\section{Modelos propuestos frente a modelo de planificación vigente}

Comparando con el modelo vigente, los modelos de planificación propuestos, por el hecho de considerar de forma más sistemática los recursos geológicos existentes, permitirían el aprovechamiento de más recursos, a pesar de su elevado nivel de exigencia, lo que garantizaría una oferta bastante prolongada en el tiempo. Aunque no es posible entrar en detalles, se evaluó también en qué medida encajarían los ámbitos extractivos del plan vigente dentro de los modelos propuestos. Se pudo comprobar que algunos de ellos deberían ser desestimados, y otros verían enormemente reducida su superficie útil. Esto es así debido a un nivel de respeto mayor de los elementos valiosos del medio natural y rural, así como de las áreas urbanas, según los criterios que se han ido exponiendo en páginas anteriores. Además, la eliminación de alguno de los ámbitos actuales, o la aplicación de mayores restricciones y condicionantes, sobre todo en los destinados al recurso más consumido (áridos para la construcción) podría dar lugar a la necesidad de búsqueda de fuentes alternativas dispersas en plazo no muy lejano, resultando un efecto de diseminación contrario al originalmente deseado. La falta de consideración de los recursos geológico-mineros existentes se presenta como un defecto importante del modelo vigente, puesto que posibilita que los recursos de áridos queden en situación de riesgo de esterilización derivado de la expansión de otros posibles usos del suelo.

\section{Conclusiones}

Se han descrito y aplicado dos alternativas de planificación territorial a la minería de áridos en la Isla de Tenerife, llamados modelo zonificado y modelo zonificado-concentrado. Las propuestas alternativas se han generado mediante el empleo de una metodología de ordenación minero-ambiental sustentada en un análisis territorial, teniendo en cuenta los valores ambientales, los efectos sobre la población y, por supuesto, los recursos mineros. Se ha comprobado que, los dos modelos propuestos proporcionan un elevado nivel de protección de los elementos más valiosos del territorio, al tiempo que se minimiza la posibilidad de conflictos. Aun así, las áreas seleccionadas como de mayor capacidad de acogida garantizan una prolongada disponibilidad temporal de recursos, lo que se debe a la importancia prestada a la distribución de los recursos geológico-mineros. Por ello, ambas fórmulas de planificación territorial permitirían revisar el modelo actual, o proporcionar un nuevo modelo de ordenación territorial de la actividad extractiva en la Isla de Tenerife. Se concluye que el conocimiento de la distribución territorial de los recursos mineros es clave para alcanzar la integración 
efectiva de la minería en la planificación, y la mejor forma posible para salvaguardarlos frente a actividades que pueden comprometer su disponibilidad futura. Esta debe ser la base de la ordenación de la actividad extractiva. Las metodologías descritas en este trabajo pueden hacerse extensivas a otros territorios, en particular insulares, en los que es muy posible que exista una problemática semejante a la existente en la isla de Tenerife.

\section{Agradecimientos}

El material que ha servido de base para la elaboración de este este artículo es fruto de un trabajo que fue realizado originalmente para el Cabildo Insular de la Isla de Tenerife por la empresa INTERRA.

\section{Referencias}

Arranz González, J.C. y Alberruche del Campo, E. 2008. Minería, medio ambiente y territorio. Monografías del Máster Internacional Aprovechamiento sostenible de los recursos minerales. UE/Programa Alfa II-0459-FA. Red DESIR. Universidad Politécnica de Madrid, Madrid. 96pp.

Arranz González, J.C., Alberruche del Campo, E. y Trapote Redondo, M.M. 2009. Metodología para la ordenación minero-ambiental de los recursos de áridos naturales. Aplicación a la ribera occidental del Ebro en Navarra. II Congreso Nacional de Áridos, Valencia, 491-495.

Ayala, F.J., Alfonso de Molina, F., Gazapo, C, de la Puente, P. y Santos, A. 1986. Bases para la ordenación minera y ambiental de la extracción de picón en las Canarias (Tenerife, Lanzarote y Gran Canaria). Publicaciones del IGME, Madrid, 103 p. y 3 mapas.

Barettino, D. 2002. Ordenación Minero-Ambiental de recursos de rocas industriales. Aplicación a la reserva estatal de pizarras de La Cabrera (León). Tesis Doctoral Universidad Politécnica de Madrid. Escuela Técnica Superior de Ingenieros de Minas. Inédito. 267 pp.

Barettino, D., Arranz-González, J.C., Martínez-Plédel, B. y Alberruche, E. 1994. Ordenación Minero-Ambiental del yacimento de pizarra de La Cabrera (León). IX Congreso Internacional de Minería y Metalurgia, León, Tomo I, 509-532.

Barettino, D., Martínez Plédel, B., Arranz-González, J.C. y Alberruche, E. 2003. Las bases para la integración de los recursos minerales en la ordenación del territorio: el Mapa de Ordenación Minero-Ambiental. In: Martins, L. y Carrión, P. (eds.). Integración de la Minería en la Ordenación del Territorio. Centro de Investigación Científica y Tecnológica, Guayaquil, 139-152.

Bellido Mulas, F., Gómez Sainz de Aja, J.A. y Barrera, J.L. 2011. Mapa Geológico Digital continuo E. 1:50000, Zona Canarias - Tenerife. (Zona-2912). In GEODE.
Mapa Geológico Digital continuo de España.[en línea]. Sistema de Información Geológica Continua: SIGECO. IGME. Editor: J. Navas [Fecha de consulta: 20/10/2011]. Disponible en: http://cuarzo.igme.es/sigeco/default.htm BOC. 2000. Decreto Legislativo $1 / 2000$ de 8 de mayo, por el que se aprueba el Texto Refundido de las Leyes de Ordenación del Territorio de Canarias y de Espacios Naturales de Canarias. Boletín Oficial de Canarias, de 15 de mayo de 2000 , no. $60,5989-6307$.

BOC. 2002. Decreto $150 / 2002$, de 16 de octubre, por el que se aprueba definitivamente el Plan Insular de Ordenación de Tenerife. Boletín Oficial de Canarias, de 19 de octubre de 2002, no. 140, 17206-17431.

BOC. 2010. Decreto 174/2009, de 29 de diciembre, por el que se declaran Zonas Especiales de Conservación integrantes de la Red Natura 2000 en Canarias y medidas para el mantenimiento en un estado de conservación favorable de estos espacios naturales. Boletín Oficial de Canarias, de 13 de enero de 2010, no. 7, 592-2165.

BOC. 2011. Decreto 56/2011, de 4 de marzo, por el que se aprueba la Revisión Parcial del Plan Insular de Ordenación de Tenerife (PIOT) para su adaptación a las Directrices de Ordenación General, para la racionalización del planeamiento territorial de desarrollo del PIOT y para la puesta de manifiesto de la complementariedad de las infraestructuras portuarias insulares. Boletín Oficial de Canarias, de 21 de marzo de 2011, no. 58, 5440-5822.

Carracedo, J.C. 1994. The Canary Islands: an example of structural control growth of large oceanic-island volcanoes. Journal of Volcanology and Geothermal Research, 60, 225-261.

Carracedo, J.C. 2008. El Teide y las dorsales de Tenerife: un sistema volcánico interactivo. Excursión pre-congreso n 6. In: Pérez-Torrado, F.J. y Cabrera, M.C. (eds.). GeoGuías 4, pp. 155-181.

Gómez Orea, D. 1994. Ordenación del territorio: una aproximación desde el medio físico. Instituto Tecnológico Geominero de España-Editorial Agrícola Española, S.A. Madrid. 238 pp.

Hernández-Durán, G. 2000. Plan especial de Ordenación de la actividad extractiva. Granadilla de Abona, Santa Cruz de Tenerife, Islas Canarias. Informe inédito. Tenerife, $134 \mathrm{pp}$.

IGN (Instituto Geográfico Nacional), 6/10/2014, http:// www2.ign.es/iberpix/visoriberpix/visorign.html

IGME. 1995. Ordenación minero-ambiental del yacimiento de pizarras ornamentales de La Cabrera (León). Informe inédito. Servicio de Documentación del IGME. Madrid. 7 tomos.

INTERRA. 2007. Mapa de compatibilidad Minero-Ambiental de La Rioja. Gobierno de La Rioja, Dirección General de Política Territorial. $241 \mathrm{pp}$.

INTERRA. 2011. Estudio de la ordenación territorial de la actividad extractiva en Tenerife. Memoria. Informe inédito. 313 pp.

ITGE. 1973. Mapa de Rocas Industriales, escala 1:200.000. Hoja y memoria 91-10-10/11 Tenerife. Servicio de Publicaciones del Ministerio de Industria, 58 pp. 
Kužvart, M. 1984. Industrial minerals and rocks. Developments in Economic Geology, vol. 18. Elsevier, Amsterdam, 454 pp.

Langer, W.H. 2002. Managing and Protecting Aggregate Resources. USGS, Open-File Report 02-415. 15 pp.

Martínez, B., Arranz, J.C. Alberruche, E. y Barettino, D. 2006. Los proyectos de ordenación minero-ambiental de las rocas y minerales industriales. Algunos casos en España. Boletín Geológico y Minero, 117 (2), 305-316.

McEvoy, F.M., Cowley, J., Hobben, K., Bee, E. y Harris, S. 2007. A guide to mineral safeguarding in England. British Geological Survey Commissioned Report, CR/07/060, 36 pp.
Ministerio de Industria Energía y Turismo, 2/10/2014, http://www.minetur.gob.es/energia/mineria/ Estadistica/Paginas/Consulta.aspx

Stenestad, E. and Sustrac, G.1994. The role of geoscience in planning and development. In: Lumsden, G.I. (ed.). Geology and the Environment in Western Europe. Oxford Academic Press, London, pp. 281-301.

Tandy, C. 1979. Industria y paisaje. Instituto de Estudios de la Administración Local. Madrid. 386 pp.

Wrighton, C.E. y Humpage, A.J. 2012. Aggregates Safeguarding Maps of Wales. British Geological Survey Commissioned Report CR/12/039, 20 pp.

Recibido: julio 2014

Revisado: noviembre 2014

Aceptado: noviembre 2014

Publicado: diciembre 2016 
